\title{
A Quantitative Study of Local Ternary Patterns for Risk Assessment in Mammography
}

\author{
Andrik Rampun $^{1 \star}$, Philip J. Morrow ${ }^{1}$, Bryan W. Scotney ${ }^{1}$, and John Winder ${ }^{2}$ \\ ${ }^{1}$ School of Computing and Information Engineering, Ulster University, Coleraine, \\ N.Ireland, BT52 1SA \\ ${ }^{2}$ School of Health Sciences, Institute of Nursing and Health, Ulster University, \\ Newtownabbey, N. Ireland, BT37 0QB \\ \{y.rampun, pj.morrow, bw.scotney, $r j$. winder\}@ulster.ac.uk
}

\begin{abstract}
This paper presents a preliminary quantitative study for breast cancer risk assessment in mammography using mathematical operators called Local Ternary Patterns. The study covers three different mapping patterns namely uniform (' $u 2$ '), nonuniform (' $r i$ ') and a combination of uniform and nonuniform ('riu2'). These patterns are used as texture features to model the appearance of breast density within the fibroglandular disk area. Subsequently, the Support Vector Machine is employed as a classification approach and initial results suggest that the mapping pattern 'riu2' outperforms the others.
\end{abstract}

Keywords: Computer Aided Diagnosis, Local Ternary Patterns, Breast Cancer and Mammography

\section{Introduction}

The use of Computer Aided Diagnosis (CAD) systems as a second reader opinion is increasingly popular in medical applications as it helps radiologists to interpret images efficiently and to produce results consistently. One of the elements that determines the reliability of a CAD system is the use of robust descriptors to capture distinctive characteristics of the region of interest in an image. Many texture descriptors have been investigated in the literature for breast density classification in mammograms. The most popular are based on the first and second-order statistical features due to its simplicity. Texture descriptors such as local binary patterns (LBP) and textons are also quite popular due to their ability to capture rich and descriptive characteristics of the texture. We refer the reader to the study in [1] for full reviews of existing methods in the literature. Our study is motivated from the study of Hadid et al. [3]. They found that at least $80 \%$ of the textures in natural images are dominated by uniform patterns, hence suggesting that the mapping pattern ' $u 2$ ' is the most reliable in capturing the characteristics of natural images. We are interested to know as to whether their findings are the same in mammogram breast density classification

\footnotetext{
* Corresponding author
} 


\section{Rampun et al.}

problems. Therefore, we study the other mapping patterns ('ri' and 'riu2'). To our knowledge, none of the existing studies have investigated the use of Local Ternary Patterns for breast density classification in mammography.

\section{Methodology}

We separate the breast region from the background and pectoral muscle, and extract only the fibroglandular disk area $\left(F G D_{\text {roi }}\right)$. Subsequently, we use a simple median filter for noise reduction and extract Local Ternary Patterns (LTPs) to capture the micro-structure information of $F G D_{\text {roi }}$ (see Figure 1). Finally, we train a SVM classifier and used the model to test unseen cases.

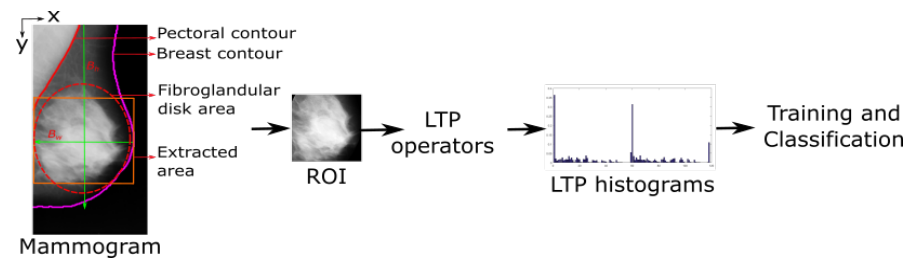

Fig. 1. An overview of the feature extraction on $F G D_{\text {roi }}$.

\subsection{Local Ternary Patterns}

The LTP thresholds the neighbouring pixels into three values $-1,0$ and 1 based on the threshold value $(k)$ set by the user. It has the following parameters: $\theta$ (orientation), $k$ (threshold value set by the user), $R$ (radius) and $P$ (number of neighbours). The LTP decimal value of a pixel $(i, j)$ is given by:

$$
\operatorname{LTP}_{(P, R)}^{\text {pattern }}(i, j)=\sum_{p=0}^{(P-1)} s_{\text {pattern }}\left(g_{p}\right) 2^{p}
$$

where $R$ is the circle radius, $P$ is the number of neighbours, $k$ is the threshold constant, $g_{c}$ is the grey level value of the center pixel, $p$ is the neighbouring pixel, $g_{p}$ is the grey level value of the $p^{t h}$ neighbour, and pattern $\in\{$ upper,lower $\}$. Once the LTP code is generated, it is split into two binary patterns (upper and lower patterns) by considering its positive, zero and negative components, using the following conditions

$$
\begin{aligned}
& s_{\text {upper }}(p)= \begin{cases}1, & \text { if } s(p)>0 \\
0, & \text { if } s(p) \leq 0\end{cases} \\
& s_{\text {lower }}(p)= \begin{cases}1, & \text { if } s(p)<0 \\
0, & \text { if } s(p) \geq 0\end{cases}
\end{aligned}
$$


The LTP code can be generated using the following conditions

$$
s(p)= \begin{cases}-1, & \text { if } p<g_{c}-k \\ 0, & \text { if } p \geq g_{c}-k \text { and } p \leq g_{c}+k \\ 1, & \text { if } p>g_{c}+k\end{cases}
$$

where $s(p)$ is the $p^{\text {th }}$ neighbour containing the LTP code value. Figure 2 shows an illustration of different LTP patterns namely ' $u 2$ ', ' $r i$ ' and 'riu2'. Note that the black dots are neighbours with higher values than its central pixels (red dot).

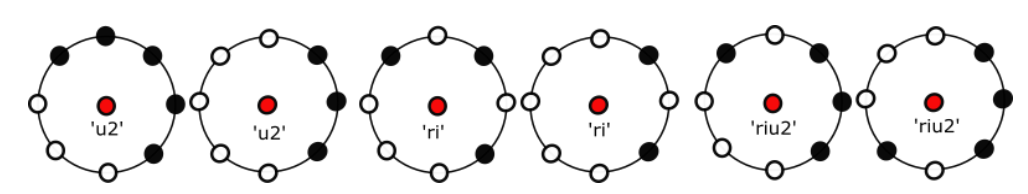

Fig. 2. An illustration of different LTP patterns with $P=8$ and $R=3$.

\section{Experimental Results}

The study was conducted based on the well known Mammographic Image Analysis Society (MIAS) database [2] which consists of 322 mammograms of 161 women. Each image contains BIRADS information (e.g. BIRADS class I, II, III or IV) provided by an expert radiologist (class I and II and class III and IV are considered as low and high risk, respectively). A stratified ten runs 10-fold cross validation scheme was employed, where the patients are randomly split into $90 \%$ for training and $10 \%$ for testing and repeated 100 times.

Table 1 presents the quantitative results of two-class classification when using different mapping patterns with different parameters values. Since this paper presents our preliminary investigation, at this stage we have set $k=4$ and $\theta=0$ for all experiments. The results indicate that the the LTP operators produce more discriminative features when the mapping pattern 'riu2' is used to model the appearance of the $F G D_{\text {roi }}$. The mapping pattern ' $r i$ ' produced the second best result on average across different parameter settings whereas the pattern ' $u 2$ ' is slightly below the performance of the other patterns. Overall, we would like to highlight the following findings: (a) the ' $r i$ ' mapping pattern performed better at smaller $R$ compared to both ' 42 ' and 'riu2', (b) using a medium size of $R$ tends to produce higher accuracies for both ' $u 2$ ' and 'riu2', (c) the 'riu2' mapping pattern produced consistent results when a larger number of neighbours $(P)$ is used and $(\mathrm{d})$ all mapping patterns are quite dependent on the parameter values. 
Table 1. Quantitative results for two-class classification problem using different parameters and mapping patterns. We set $k=4$ and $\theta=0$ for all experiments

\begin{tabular}{|c|c|c|c|c|}
\hline \multirow{2}{*}{$P$} & \multirow{2}{*}{$R$} & \multicolumn{3}{|c|}{ Accuracy(\%) } \\
\cline { 3 - 5 } & & 'u2' & 'ri' & 'riu2' \\
\hline 8 & 2 & 84.56 & 87.75 & 84.71 \\
\hline 12 & 3 & 84.78 & 86.84 & 88.59 \\
\hline 6 & 3 & 87.31 & $\mathbf{8 9 . 3 4}$ & 86.00 \\
\hline 6 & 5 & 87.56 & 88.81 & 88.78 \\
\hline 16 & 5 & $\mathbf{8 9 . 0 3}$ & 85.45 & 89.65 \\
\hline 12 & 5 & 88.03 & 84.59 & 89.65 \\
\hline 18 & 7 & 88.68 & 86.23 & $\mathbf{9 0 . 8 4}$ \\
\hline 12 & 7 & 87.68 & 85.18 & 90.25 \\
\hline 8 & 7 & 88.93 & 87.93 & 89.31 \\
\hline 18 & 9 & 87.53 & 86.68 & 89.53 \\
\hline 14 & 9 & 86.53 & 85.00 & 89.56 \\
\hline 10 & 9 & 85.84 & 89.21 & 90.31 \\
\hline 8 & 9 & 85.56 & 89.15 & 89.62 \\
\hline
\end{tabular}

\section{Conclusions}

Our experimental results indicate that in the mammogram breast density classification problem, using the 'riu2' mapping pattern is more suitable than using ' $r i$ ' and ' $u 2$ '. Our findings are different from the study of Hadid et al. [3] due to differences in datasets (e.g. their dataset contains images with distinctive and visible texture patterns whereas most breast regions contain blurred and diffused texture patterns which make it more difficult to model the appearance). In future work, we are interested in combining the $L T P^{\text {riu2 }}$ features with other texture descriptors and investigate other parameters such as $k$ and $\theta$.

Acknowledgments. This research was undertaken as part of the Decision Support and Information Management System for Breast Cancer (DESIREE) project. The project has received funding from the European Union's Horizon 2020 research and innovation programme under grant agreement No 690238.

\section{References}

1. A. Oliveira, J. A Fonseca-Moutinho, M. Pereira and M. M. Freire. A survey of the methods used to classify breast density in mammograms and ultrasound images [Online]. Available: http://www.di.ubi.pt/ mario/Angela1.pdf. [Accessed:3-Feb-2017].

2. J. Suckling et al.. The mammographic image analysis society digital mammogram database. In Proc. Excerpta Med. Int. Congr. Ser., pp. 375-378, 1994.

3. A. Hadid, M. K. Pietikainen, G. Zhao and T. Ahonen. Computer Vision Using Local Binary Patterns, Springer London, pp. 13-47, 2011. 